\title{
COMPLETE METRIC ON MIXING ACTIONS OF GENERAL GROUPS
}

\author{
TIKHONOV S.V.
}

\begin{abstract}
In this paper the metric on the set of mixing actions of a countable infinite group is introduced so that the corresponding space is complete and separable.
\end{abstract}

\section{INTRODUCTION}

Let $\mathcal{A}$ be the group of invertible measure preserving transformations of Lebesgue space $(X, \Sigma, \mu)$, endowed with weak topology(see below). Continuous homomorphism of a topological group $\mathcal{G}$ into $\mathcal{A}$ is called $\mathcal{G}$-action(or simply, action).

Measure preserving action $T$ of a locally compact group is mixing if for arbitrary measurable sets $A$ and $B \subset X$

$$
\mu\left(T^{g} A \cap B\right) \rightarrow \mu(A) \mu(B) \text { as } g \rightarrow \infty ;
$$

if $\mathcal{G}$ is finitely generated then one can say about length of its elements, denoted by $|\bullet|$, and $g \rightarrow \infty$ iff $|g| \rightarrow \infty$.

The aim of the paper is to elaborate certain machinery for study of mixing $\mathcal{G}$-actions from the point of view going back to the well-known theorem due to Halmos and Rokhlin about conjugacy class of aperiodic transformation.

This question for $\mathbb{Z}$-actions was considered in [1].

Here the general case of countable infinite groups and their actions is considered:

- the metric on the set of mixing $\mathcal{G}$-actions is introduced so that the corresponding space $\mathcal{M}_{\mathcal{G}}$ is complete and separable,

- it is shown that for generic action its conjugates are everywhere dense in this space.

Moreover, if $\mathcal{G}$ is finitely generated infinite monotilable amenable group then conjugates to Cartesian product of two free mixing actions are everywhere dense in $\mathcal{M}_{\mathcal{G}}$.

\section{THE METRIC}

Let $(X, \Sigma, \mu)$ be separable Lebesgue space and $\left\{A_{i}\right\}$ be a countable collection of sets generating the $\sigma$-algebra $\Sigma$. Measure $\mu$ is supposed to be normalized and continuous. All the spaces with these properties are isomorphic to each other; in particular such a space is isomorphic to its Cartesian square. The set $\mathcal{A}$ of invertible measure preserving transformations $X$ is separable with respect to weak topology defined by the base of neighbourhoods

$$
\mathcal{U}(T, q, \varepsilon)=\{S|\forall A, B \in q:| \mu(T A \cap B)-\mu(S A \cap B) \mid<\varepsilon\},
$$

(here and below $q$ stand for finite subset of $\left\{A_{i}\right\}$ ). Moreover $\mathcal{A}$ is a topological group (i.e. multiplication and inversion are continuous). The weak topology is generated by any of the metrics

$$
d(T, S)=\sum_{i \in \mathbb{N}} \frac{1}{2^{i}}\left(\mu\left(T A_{i} \triangle S A_{i}\right)+\mu\left(T^{-1} A_{i} \triangle S^{-1} A_{i}\right)\right),
$$

and

$$
a(T, S)=\sum_{i, j \in \mathbb{N}} \frac{1}{2^{i+j}}\left|\mu\left(T A_{i} \cap A_{j}\right)-\mu\left(S A_{i} \cap A_{j}\right)\right| .
$$

Key words and phrases. monotilable group and measure preserving transformations and mixing group actions. 
Metric $d$ is more preferable in the sense that the space $\mathcal{A}$ is complete 1 with respect to $d$; it is called metric of weak topology. This metric can be naturally generalized to the case of group actions. Let $\mathcal{G}$ be countable group; the set $\mathcal{A}_{\mathcal{G}}$ of $\mathcal{G}$-actions by measure preserving transformations is a complete separable metric space with respect to metric

$$
d(T, S)=\sum_{g \in \mathcal{G}} \frac{1}{2^{|g|}} d\left(T^{g}, S^{g}\right),
$$

where $\{|g|\}_{g \in \mathcal{G}}$ is collection of positive numbers satisfying the condition $\sum_{g \in \mathcal{G}} \frac{1}{2^{|g|}}<\infty$.

Action $T$ of a group $\mathcal{G}$ is called mixing, if for each pair $A, B \in \Sigma$, one has

$$
\mu\left(T^{g} A \cap B\right) \rightarrow \mu(A) \mu(B)
$$

as $|g| \rightarrow \infty$.

The set $\mathcal{M}_{\mathcal{G}}$ of mixing actions of $\mathcal{G}$ is endowed by lead metric

$$
m(T, S)=d(T, S)+\sup _{g \in \mathcal{G}} a\left(T^{g}, S^{g}\right) .
$$

If $\mathcal{G}=\mathbb{Z}$ the metric $m$ coincides with complete separable metric introduced in [1]. Metric $m(T, S)$ can be considered on the set $\mathcal{A}_{\mathcal{G}}$, however it is not separable (even in the case $\mathcal{G}=\mathbb{Z}$ ).

Lemma 1. Metrics $m$ and $w, w=\sup _{g \in \mathcal{G}} a\left(T^{g}, S^{g}\right)$ generate in $\mathcal{M}_{\mathcal{G}}$ the same topology with the base of neighbourhoods

$$
\mathcal{Q}(T, q, \varepsilon)=\left\{S\left|\forall A, B \in q, \sup _{g \in \mathcal{G}}\right| \mu\left(T^{g} A \cap B\right)-\mu\left(S^{g} A \cap B\right) \mid<\varepsilon\right\},
$$

where $T \in \mathcal{M}_{\mathcal{G}}, q \subset\left\{A_{i}\right\}$ is finite and $\varepsilon \in \mathbb{R}^{+} \backslash\{0\}$.

Proof. Coincidence of topologies follows from the fact that metrics $d$ and $a$ generate the same topology (see [1]). Now we show that sets $\mathcal{Q}(T, q, \varepsilon)$ form a base of topology generated by $w$.

1. In every $(w, \delta)$-neighbourhood of the action $T$ there is a neighbourhood $\mathcal{Q}\left(T, q, \frac{\delta}{4}\right)$, where $q$ is such that $2 \sum_{A_{i} \notin q} \frac{1}{2^{i}}<\frac{\delta}{2}$. In fact, one has

$$
\begin{gathered}
S \in \mathcal{Q}\left(T, q, \frac{\delta}{4}\right) \Rightarrow \forall g: a\left(T^{g}, S^{g}\right)=\sum_{i, j \in \mathbb{N}} \frac{1}{2^{i+j}}\left|\mu\left(T^{g} A_{i} \cap A_{j}\right)-\mu\left(S^{g} A_{i} \cap A_{j}\right)\right| \leqslant \\
\leqslant \sum_{A_{i}, A_{j} \in q} \frac{1}{2^{i+j}}\left|\mu\left(T^{g} A_{i} \cap A_{j}\right)-\mu\left(S^{g} A_{i} \cap A_{j}\right)\right|+\sum_{A_{i} \notin q} \frac{1}{2^{i}}+\sum_{A_{j} \notin q} \frac{1}{2^{j}} \leqslant \\
\leqslant \sum_{A_{i}, A_{j} \in q} \frac{1}{2^{i+j}}\left|\mu\left(T^{g} A_{i} \cap A_{j}\right)-\mu\left(S^{g} A_{i} \cap A_{j}\right)\right|+\frac{\delta}{2} \leqslant \frac{3 \delta}{4} .
\end{gathered}
$$

Since this is true for any $g$,

$$
w(T, S)=\sup _{g \in \mathcal{G}} a\left(T^{g}, S^{g}\right)<\delta .
$$

2. In every set $\mathcal{Q}(T, q, \varepsilon)$ there is $\left(w, \frac{\varepsilon}{2^{2 l}}\right)$-neighbourhood of $T$, where $l$ is the maximal number of elements from $q$. Then for $A_{i}, A_{j} \in q$, one has

$$
\begin{gathered}
\frac{1}{2^{2 l}}\left|\mu\left(S^{g} A_{i} \cap A_{j}\right)-\mu\left(T^{g} A_{i} \cap A_{j}\right)\right| \leqslant \frac{1}{2^{i+j}}\left|\mu\left(S^{g} A_{i} \cap A_{j}\right)-\mu\left(T^{g} A_{i} \cap A_{j}\right)\right| \leqslant \\
\leqslant \sum_{i, j \in \mathbb{N}} \frac{1}{2^{i+j}}\left|\mu\left(S^{g} A_{i} \cap A_{j}\right)-\mu\left(T^{g} A_{i} \cap A_{j}\right)\right|=a\left(S^{g}, T^{g}\right) .
\end{gathered}
$$

\footnotetext{
${ }^{1}$ To speak about completeness it is necessary to identify transformations (or $\mathcal{G}$-actions that will be the main object below), coinciding mod 0 .
} 
Hence for each $S$ such that $w(T, S)<\frac{\varepsilon}{2^{2 l}}$, every pair $A, B \in q$ and every $g \in \mathcal{G}$ the inequality

$$
\sup _{g \in \mathcal{G}}\left|\mu\left(T^{g} A \cap B\right)-\mu\left(S^{g} A \cap B\right)\right| \leqslant 2^{2 l} a\left(S^{g}, T^{g}\right)<\varepsilon,
$$

holds and therefore $S \in \mathcal{Q}(T, q, \varepsilon)$.

Lemma 2. The space $\mathcal{M}_{\mathcal{G}}$ is separable.

Proof. It is sufficient to show that for each finite $q \subset\left\{A_{i}\right\}$ and arbitrary $\varepsilon>0$ one can choose the countable sets of actions $\left\{T_{l}\right\}$ such that the space $\mathcal{M}_{\mathcal{G}}$ has a covering by the sets $\left\{\mathcal{Q}\left(T_{l}, q, \varepsilon\right)\right\}_{l}$.

For every mixing $\mathcal{G}$-action $T$ there is a finite collection of parameters defined as follows:

$n$ is a positive integer such that

$$
A, B \in q,|g|>n \Rightarrow\left|\mu\left(T^{g} A \cap B\right)-\mu(A) \mu(B)\right|<\frac{\varepsilon}{2}
$$

$\left\{n_{g}\right\}_{|g| \leqslant n}$ is the collection of positive integers such that

$$
A, B \in q,|g| \leqslant n \Rightarrow\left|\mu\left(T^{g} A \cap B\right)-\frac{\varepsilon n_{g}}{2}\right|<\frac{\varepsilon}{2} .
$$

The set of these parameters is countable. If actions $T$ and $S$ have the same parameter collections then for each pair $A, B \in q$, one has

$$
\begin{gathered}
\sup _{g}\left|\mu\left(T^{g} A \cap B\right)-\mu\left(S^{g} A \cap B\right)\right|= \\
=\max \left\{\sup _{|g| \leqslant n}\left|\mu\left(T^{g} A \cap B\right)-\mu\left(S^{g} A \cap B\right)\right|, \sup _{|g|>n}\left|\mu\left(T^{g} A \cap B\right)-\mu\left(S^{g} A \cap B\right)\right|\right\} \leqslant \\
\leqslant \max \left\{\sup _{|g| \leqslant n}\left(\left|\mu\left(T^{g} A \cap B\right)-\frac{\varepsilon n_{g}}{2}\right|+\left|\mu\left(S^{g} A \cap B\right)-\frac{\varepsilon n_{g}}{2}\right|\right),\right. \\
\left.\sup _{|g|>n}\left(\left|\mu\left(T^{g} A \cap B\right)-\mu(A) \mu(B)\right|+\left|\mu(A) \mu(B)-\mu\left(S^{g} A \cap B\right)\right|\right)\right\}<\varepsilon .
\end{gathered}
$$

Hence $S \in \mathcal{Q}(T, q, \varepsilon)$ and the required countable set of actions can be formed by choosing one action for each of the constructed parameter collections.

Theorem 1. $\mathcal{M}_{\mathcal{G}}$ is a complete separable metric space.

Proof. By virtue of the previous lemma it remains to check completeness. Let $\left\{T_{i}\right\} \subset \mathcal{M}_{\mathcal{G}}$ be a Cauchy sequence with respect to $m$. Then it is also Cauchy in the space $\mathcal{A}_{\mathcal{G}}$ and converges in $\mathcal{A}_{\mathcal{G}}$ to some $\mathcal{G}$-action $T$. In addition $T_{i}^{g} \rightarrow T^{g}$ for every $g \in \mathcal{G}$. Since $d\left(T, T_{i}\right) \rightarrow 0$ it is sufficient to check that $T$ is mixing and

$$
\sup _{g} a\left(T^{g}, T_{i}^{g}\right) \rightarrow 0
$$

Let $i \in \mathbb{N}$ be such that for any $l, m>i$ and every $g \in \mathcal{G}$ one has $a\left(T_{l}^{g}, T_{m}^{g}\right)<\varepsilon$. Now $T_{l}^{g}$ converges weakly to $T^{g}$, so there exists $n(g)>i$ such that $a\left(T_{n(g)}^{g}, T^{g}\right)<\varepsilon$.

It follows that for $m>i$,

$$
\sup _{g} a\left(T^{g}, T_{m}^{g}\right) \leqslant \sup _{g}\left(a\left(T^{g}, T_{n(g)}^{g}\right)+a\left(T_{m}^{g}, T_{n(g)}^{g}\right)\right)<2 \varepsilon .
$$

Next, for $m$ chosen above and given sets $A_{i}, A_{j}$ there is a number $k_{0}$ such that

$$
\left|\mu\left(T_{m}^{g} A_{i} \cap A_{j}\right)-\mu\left(A_{i}\right) \mu\left(A_{j}\right)\right|<\varepsilon,
$$

if $|g|>k_{0}$.

Now, using the inequality

$$
\frac{1}{2^{i+j}}\left|\mu\left(T_{m}^{g} A_{i} \cap A_{j}\right)-\mu\left(T^{g} A_{i} \cap A_{j}\right)\right| \leqslant
$$




$$
\leqslant \sum_{i, j \in \mathbb{N}} \frac{1}{2^{i+j}}\left|\mu\left(T_{m}^{g} A_{i} \cap A_{j}\right)-\mu\left(T^{g} A_{i} \cap A_{j}\right)\right|=a\left(T_{m}^{g}, T^{g}\right)
$$

one has

$$
\begin{gathered}
\left|\mu\left(T^{g} A_{i} \cap A_{j}\right)-\mu\left(A_{i}\right) \mu\left(A_{j}\right)\right| \leqslant \\
\leqslant\left|\mu\left(T_{m}^{g} A_{i} \cap A_{j}\right)-\mu\left(T^{g} A_{i} \cap A_{j}\right)\right|+\left|\mu\left(A_{i}\right) \mu\left(A_{j}\right)-\mu\left(T_{m}^{g} A_{i} \cap A_{j}\right)\right|< \\
<\varepsilon\left(2^{i+j+1}+1\right),
\end{gathered}
$$

whence in view of arbitrariness of $\varepsilon, i$ and $j$ it follows that $T$ is mixing.

\section{Dense subsets}

We call the family of actions

$$
\left\{U^{-1} T U\right\}_{U \in \mathcal{A}}
$$

conjugacy class of $\mathcal{G}$-action $T$. The closure of this class in $\mathcal{M}_{\mathcal{G}}$ is denoted by $\bar{T}$.

Lemma 3. For each $U \in \mathcal{A}$ the mapping $\widetilde{U}: T \mapsto U^{-1} T U, \mathcal{M}_{\mathcal{G}} \rightarrow \mathcal{M}_{\mathcal{G}}$ is continuous.

Proof. Consider neighbourhoods $\mathcal{Q}\left(U^{-1} T U, q, \varepsilon\right)$ and $\mathcal{Q}(T, U q, \varepsilon)$ of the actions $U^{-1} T U$ and $T$ respectively.

Then

$$
\begin{gathered}
P \in \mathcal{Q}(T, U q, \varepsilon) \Rightarrow \\
\Rightarrow \forall A, B \in q: \varepsilon>\sup _{g \in \mathcal{G}}\left|\mu\left(T^{g} U A \cap U B\right)-\mu\left(P^{g} U A \cap U B\right)\right|= \\
=\sup _{g \in \mathcal{G}}\left|\mu\left(U^{-1} T^{g} U A \cap B\right)-\mu\left(U^{-1} P^{g} U A \cap B\right)\right| \Rightarrow \\
\Rightarrow U^{-1} P U \in \mathcal{Q}\left(U^{-1} T U, q, \varepsilon\right) .
\end{gathered}
$$

The $\mathcal{G}$-action $S$ is a factor of $\mathcal{G}$-action $T$, if there exists measure preserving mapping $v$ of the space $(X, \Sigma, \mu)$ into itself such that $v \circ T^{g}=S^{g} \circ v \bmod 0$ for every $g \in \mathcal{G}$.

Lemma 4. If $S$ is a factor of $T$ then $\bar{S} \subset \bar{T}$.

Proof. It suffices to check that $S \in \bar{T}$. Let $v$ be so that $v \circ T^{g}=S^{g} \circ v \bmod 0$. Consider a neighbourhood $\mathcal{Q}(S, q, \varepsilon)$ of the action $S$ and show that in this neighbourhood there is an action of the form $U^{-1} T U$ for certain $U \in \mathcal{A}$. We take as $U$ any invertible measure preserving transformation of the space $(X, \Sigma, \mu)$ transferring $A$ to $v^{-1} A$ for each $A \in q$.

Then for all $g \in \mathcal{G}, A, B \in q$, we have

$$
\begin{gathered}
\mu\left(S^{g} A \cap B\right)-\mu\left(U^{-1} T^{g} U A \cap B\right)=\mu\left(S^{g} A \cap B\right)-\mu\left(T^{g} U A \cap U B\right)= \\
=\mu\left(S^{g} v v^{-1} A \cap B\right)-\mu\left(T^{g} v^{-1} A \cap v^{-1} B\right)= \\
=\mu\left(v^{-1} S^{g} v v^{-1} A \cap v^{-1} B\right)-\mu\left(T^{g} v^{-1} A \cap v^{-1} B\right)= \\
=\mu\left(v^{-1} v T^{g} v^{-1} A \cap v^{-1} B\right)-\mu\left(T^{g} v^{-1} A \cap v^{-1} B\right)=0 .
\end{gathered}
$$

The last equality follows from the inclusion $v^{-1} v T^{g} v^{-1} A \supset T^{g} v^{-1} A$ and the fact that measures of these sets coincide, hence they are equal mod 0 (it is used here that $\mu\left(v^{-1} v T^{g} v^{-1} A\right)=\mu\left(v^{-1} S^{g} A\right)=$ $\mu(A))$.

Corollary 1. Actions $T$ and $S$ can be approximated arbitrarily well by actions conjugate to $T \times S$.

Subset of metric space has type $G_{\delta}$ if it is countable intersection of open sets. Everywhere dense subset in a complete metric space is called generic. The expression "typical mixing possesses the property $P$ " means that this $P$ hold for generic set of mixing actions. 
Theorem 2. The set

$$
W=\left\{T \in \mathcal{M}_{\mathcal{G}} \mid \bar{T}=\mathcal{M}_{\mathcal{G}}\right\}
$$

is generic.

Proof. Let $\left\{T_{i}\right\}$ be a countable dense subset in $\mathcal{M}_{\mathcal{G}}$. The set $W$ consists of those mixing actions that can be transferred by conjugation into each of the sets

$$
\mathcal{U}_{n}\left(T_{i}\right)=\left\{S \mid m\left(T_{i}, S\right)<\frac{1}{n}\right\}, \quad i, n \in \mathbb{N} .
$$

Thus

$$
W=\bigcap_{i, n}\left\{S \mid \exists U: U^{-1} S U \in \mathcal{U}_{n}\left(T_{i}\right)\right\}
$$

Note that all the sets right to the intersection symbol are open. Indeed, $\widetilde{U}(T): T \mapsto U^{-1} T U$ is continuous mapping, hence $\widetilde{U}$ - inverse image of a neighbourhood of the action $U^{-1} S U$ contains some neighbourhood of $S$.

It remains to note that conjugacy class for Cartesian product of all $T_{i}$ 's is dense in $\mathcal{M}_{\mathcal{G}}$, since it approaches arbitrarily close to each of $T_{i}{ }^{\text {}} \mathrm{s}(\operatorname{cor}$ 1).

\section{EvERYWHERE DENSE SETS OF ACTIONS FOR MONOTILABLE AMENABLE GROUPS}

In the section we consider finitely generated infinite monotilable amenable groups.

Definition 1. Group $\mathcal{G}$ is called monotilable amenable, if there exists a sequence $\left\{F_{i}\right\}_{i \in \mathbb{N}}$ of finite subsets in $\mathcal{G}$, satisfying the following conditions:

1. For every $g \in \mathcal{G}$, one has $\frac{\#\left(g F_{i} \cap F_{i}\right)}{\# F_{i}} \rightarrow 1$ as $i \rightarrow \infty$ (here and then symbol "\#" denotes the number of element in the set);

2. For each $i \in \mathbb{N}$, the set $F_{i}$ is a tile in the sense that there exists a collection $\left\{c_{j}^{(i)}\right\} \subset \mathcal{G}$ such that $\mathcal{G}=\bigsqcup_{j} F_{i} c_{j}^{(i)}$

We recall that an action $T$ is free if the set

$$
\left\{x \in X \mid \exists g \neq e, T^{g} x=x\right\}
$$

has measure zero.

The following proposition [2] is a group analog of Rokhlin-Halmos lemma.

Lemma 5. For arbitrary $\varepsilon>0$, free $\mathcal{G}$-action $Q$ and a tile $G \subset \mathcal{G}$, there exists a set $E$ such that $Q^{g} E, g \in G$ are disjoint and their total measure in not less $1-\varepsilon$.

A collection of sets $\left\{Q^{g} E\right\}_{g \in G}$ with $E$ as in lemma 5 is called Rokhlin tower. To mark that its remainder $O=X \backslash\left(\cup_{g \in G} Q^{g} E\right)$ has measure at most $\varepsilon$ we write $\left\{Q^{g} E\right\}_{g \in G}^{\varepsilon}$.

It follows from lemma 5 that conjugacy class of any free $\mathcal{G}$-action is everywhere dense in $\mathcal{A}_{\mathcal{G}}$.

Lemma 6. For any set $C \subset \mathcal{G}$, free $\mathcal{G}$-action $Q: Y \rightarrow Y, \varepsilon>0$ and a tile $F$ there exist sets $G \subset \mathcal{G}$ and $E \in \Sigma$ such that

1. $G=\sqcup_{i} F c_{i}$, for some finite collection $\left\{c_{i}\right\} \subset \mathcal{G}$,

2. \# $(g G \triangle G)<\varepsilon \# G$ for $g \in C$,

3. $\frac{3(\# F)^{2}}{\# G}<\varepsilon$,

4. $\left\{Q^{g} E\right\}_{g \in G}^{\varepsilon}$ is a tower.

Proof. From lemma 5 and the properties of monotilable amenable groups it follows that there exist tile $\widetilde{G}$ and set $E$ such that $\frac{3(\# F)^{2}}{\# \widetilde{G}}<\frac{\varepsilon}{2}$, \# $(g \widetilde{G} \triangle \widetilde{G})<\frac{\varepsilon}{8(\# F)^{2}} \# \widetilde{G}$ for $g \in C \cup F F^{-1}$ and $\left\{Q^{g} E\right\}_{g \in \widetilde{G}}^{\frac{\varepsilon}{2}}$ is a tower. 
Consider now an infinite sequence $\left\{b_{i}\right\}$ with the property $\mathcal{G}=\sqcup_{i} F b_{i}$ and take as $\left\{c_{i}\right\}$ the set $\left\{b_{i} \mid F b_{i} \subset \widetilde{G}\right\}$. The set $G=\sqcup_{i} F c_{i}$ possesses properties $1-4$ above.

Property 1 holds by construction of $G$.

Further,

$$
\begin{aligned}
\#(\widetilde{G} \backslash G) \leqslant \# & \left\{g \in \widetilde{G} \mid \exists f, h \in F: f^{-1} g \in\left\{b_{i}\right\}, h f^{-1} g \notin \widetilde{G}\right\} \leqslant \\
\leqslant & \sum_{f \in F F^{-1}} \#\{g \in \widetilde{G} \mid f g \notin \widetilde{G}\}< \\
& <(\# F)^{2} \frac{\varepsilon}{8(\# F)^{2}} \# \widetilde{G}=\frac{\varepsilon}{8} \# \widetilde{G},
\end{aligned}
$$

whence

$$
\#(\widetilde{G} \backslash G)<\left(\frac{1}{1-\frac{\varepsilon}{8}}-1\right) \# G<\frac{\varepsilon}{7} \# G .
$$

This estimate gives properties 3 and 4 .

For $g \in C$ one has

$$
\begin{gathered}
\#(g G \triangle G)=2 \#(g G \backslash G) \leqslant 2 \#(g \widetilde{G} \backslash \widetilde{G})+2 \#\{\widetilde{G} \backslash G\}< \\
<\frac{2 \varepsilon}{8} \# \widetilde{G}+\frac{2 \varepsilon}{7} \# G<\varepsilon \# G .
\end{gathered}
$$

Lemma 7. For arbitrary finite subsets $I, H \subset \mathcal{G}$ there exists an infinite collection $\left\{h_{i}\right\}_{1}^{\infty} \subset \mathcal{G}$ such that for every $i$

$$
h_{i}^{-1} g h_{i} \notin H \backslash\{g\}
$$

if $g \in I$.

Proof (by inductive construction). Let $H_{1} \subset \mathcal{G}$ be an infinite subset. If there are no $g \in I$ such that $h^{-1} g h \in H \backslash\{g\}$ for infinitely many $h \in H_{1}$ then the collection

$$
H_{1} \backslash \cup_{g \in I}\left\{h \mid h^{-1} g h \in H \backslash\{g\}\right\}
$$

is a required one. Otherwise there exist $g_{1} \in I$ with infinitely many inclusions $h^{-1} g_{1} h \in H \backslash\left\{g_{1}\right\}$, $h \in H_{1}$, and an infinite subset $F_{1} \subset H_{1}$ such that

$$
f_{1, i}^{-1} g_{1} f_{1, i}=f_{1, j}^{-1} g_{1} f_{1, j}
$$

for all $f_{1, i}, f_{1, j} \in F_{1}$. Hence every $h \in H_{2}:=F_{1} F_{1}^{-1}$ commutes with $g_{1}$ and thus

$$
h^{-1} g_{1} h \notin H \backslash\left\{g_{1}\right\} \text {. }
$$

If for every $g \in I \backslash\left\{g_{1}\right\}$ inclusion $h^{-1} g h \in H \backslash\{g\}$ does not hold for infinitely many $h \in H_{2}$, then

$$
H_{2} \backslash \cup_{g \in I \backslash\left\{g_{1}\right\}}\left\{h \mid h^{-1} g h \in H \backslash\{g\}\right\}
$$

is the required collection. In the case there exists $g_{2} \in I \backslash\left\{g_{1}\right\}$ with infinitely many inclusions $h^{-1} g_{2} h \in H \backslash\left\{g_{2}\right\}, h \in H_{2}$, one can choose an infinite subset $F_{2} \subset H_{2}$ such that

$$
f_{2, i}^{-1} g_{2} f_{2, i}=f_{2, j}^{-1} g_{2} f_{2, j}
$$

for all pairs $f_{2, i}, f_{2, j} \in F_{2}$. Hence every $h \in H_{3}:=F_{2} F_{2}^{-1}$ commutes with $g_{1}$ and $g_{2}$.

Doing so we either obtain the required collection or some infinite set $H_{n}$ in the centralizer of $\left\{g_{1}, \ldots, g_{n-1}\right\}$. If the set

$$
B_{n}=H_{n} \backslash \cup_{g \in I \backslash\left\{g_{1}, \ldots, g_{n-1}\right\}}\left\{h \mid h^{-1} g h \in H \backslash\{g\}\right\}
$$

is finite then $B_{n} \backslash H_{n}$ is a required collection. Otherwise there exist $g_{n} \in I \backslash\left\{g_{1}, \ldots, g_{n-1}\right\}$ with infinitely many inclusions $h^{-1} g_{n} h \in H \backslash\left\{g_{n}\right\}, h \in H_{n}$ and an infinite subset $F_{n} \subset H_{n}$ such that

$$
f_{n, i}^{-1} g_{n} f_{n, i}=f_{n, j}^{-1} g_{n} f_{n, j}
$$


for all pairs $f_{n, i}, f_{n, j} \in F_{n}$. Hence every $h \in H_{n+1}:=F_{n} F_{n}^{-1}$ commutes with $g_{1}, \ldots, g_{n}$.

Either this inductive procedure stops (whence the lemma is already proved) or infinite set $H_{\# I+1}$ (obtained as the result of the procedure) is in the centralizer of $I$ whence $H_{\# I+1}$ is a required collection.

Lemma 8. Let $\left\{C_{i}\right\}_{i \in \mathbb{N} \cup\{0\}}$ be an increasing sequence of finite subsets in $\mathcal{G}$, satisfying conditions:

$$
C_{i}=C_{i}^{-1}
$$

for all $i$ and

$$
C_{i} \backslash\left(C_{i-1}\right)^{5} \neq \varnothing
$$

for $i \in \mathbb{N}$.

Then for arbitrary collection $\left\{g_{i} \mid g_{i} \in C_{i} \backslash\left(C_{i-1}\right)^{5}\right\}$ and fixed $g \in \mathcal{G}$, there are at most 2 solutions of the inclusion

$$
g_{i} g g_{j}^{-1} \in C_{0}, i \neq j
$$

Proof. Assume that

$$
g_{i} g g_{j}^{-1}, g_{l} g g_{k}^{-1} \in C_{0} .
$$

Let $l$ be the maximal of the indices $i, j, k, l$ (other variants are considered similarly).

One has inclusions

$$
g_{i} \in C_{0} g_{j} g^{-1}, g_{l} \in C_{0} g_{k} g^{-1},
$$

and $g_{l} g_{i}^{-1} \in C_{0} g_{k} g^{-1} g g_{j}^{-1} C_{0}=C_{0} g_{k} g_{j}^{-1} C_{0}$ whence $g_{l} \in C_{0} g_{k} g_{j}^{-1} C_{0} g_{i}$. If $i, j, k \in[0, l-1]$, then $C_{0} g_{k} g_{j}^{-1} C_{0} g_{i} \subset\left(\mathrm{C}_{l-1}\right)^{5}$ in contradiction with the choice of $g_{l}$.

Hence either $l=i$ or $l=j$. If $l=i$, one has $g_{j} \in C_{0}^{-1} g_{i} g$, and on the other hand $g_{k} \in C_{0}^{-1} g_{i} g$, it follows, that $g_{j} g_{k}^{-1} \in C_{0}^{-1} C_{0}$, whence $k=j$ in view of the choice of a sequence $\left\{g_{i}\right\}$. The case $l=j$ is considered similarly and in this case $k=i$. Thus besides $\left(g_{i}, g_{j}\right)$ another solution of inclusion $g_{i} g g_{j}^{-1} \in C_{0}$ may be $\left(g_{j}, g_{i}\right)$.

Now for convenience reasons we introduce some notations. Inequality $|a-b|<\varepsilon$ will be written as proximity $a \stackrel{\varepsilon}{\sim} b$.

If an action $S$ is fixed, $A, B \in \Sigma, f, g, h \in \mathcal{G}$ we write

$$
k=f^{-1} g h, \tilde{A}_{h}=S^{h^{-1}} A, \tilde{B}_{f}=S^{f^{-1}} B .
$$

Claim 1. For $\varepsilon>0$,mixing $\mathcal{G}$-action $S$, finite $F \subset \mathcal{G}$ and finite $r \subset \Sigma$ there exists an infinite subset $\left\{g_{i}\right\} \subset \mathcal{G}$ such that given $h, f \in F$, arbitrary $A, B \in r$ and $g \in \mathcal{G}$, the proximity

$$
\mu\left(S^{g_{j}^{-1} k g_{i}} \tilde{A}_{h} \cap \tilde{B}_{f}\right) \stackrel{\varepsilon}{\sim} \mu(A) \mu(B),
$$

holds for all pairs $(i, j), i \neq j$, except at most two.

Moreover there is finite $C \subset \mathcal{G}$ such that given $h, f \in F$ arbitrary $A, B \in r$ and $g \in \mathcal{G} \backslash C$, the proximity

$$
\mu\left(S^{g_{i}^{-1} k g_{i}} \tilde{A}_{h} \cap \tilde{B}_{f}\right) \stackrel{\varepsilon}{\sim} \mu(A) \mu(B)
$$

holds for all $i$, except at most one.

Proof. Let $N$ be such that

$$
|g|>N-2 \max _{f \in F}|f| \Rightarrow \mu\left(S^{g} A \cap B\right) \stackrel{\varepsilon}{\sim} \mu(A) \mu(B)
$$

for any $A, B \in r$. Take $\{g \in \mathcal{G}|| g \mid \leqslant N\}$ as $C$ and set $H=\bigcap_{f, h \in F} f^{-1} C h$. One has that $g \notin H$ implies the proximities

$$
\forall f, h \in F, A, B \in r,: \mu\left(S^{g} \tilde{A}_{h} \cap \tilde{B}_{f}\right) \stackrel{\varepsilon}{\sim} \mu\left(\tilde{A}_{h}\right) \mu\left(\tilde{B}_{f}\right)=\mu(A) \mu(B),
$$

and moreover $C=C^{-1}$. 
The required sequence $\left\{g_{i}\right\}$ and auxiliary sequence of subsets $C_{i} \subset \mathcal{G}, g_{i} \in C_{i}$, will be constructed inductively.

We set $C_{0}=H$ and let $g_{1}$ be an arbitrary element outside $C_{0}^{5}$. As $C_{1}$ may be taken finite set, containing $g_{1}, C_{0}^{5}$ and invariant with respect to inversion $g \rightarrow g^{-1}$.

Let $g_{1}, \ldots, g_{n-1}$ and $C_{n-1}$ are already chosen. Applying lemma 7 to the sets $H$ and $I_{n}=$ $\bigcup_{i<n} g_{i} H g_{i}^{-1}$ choose elements $\left\{h_{i}\right\}$. Next as $g_{n}$ take a arbitrary element of set $\left\{h_{i}\right\} \backslash C_{n-1}^{5}$, and then as $C_{n}$ take arbitrary finite set, containing $g_{n}, C_{n-1}^{5}$ and invariant with respect to inversion.

This inductive procedure results in the increasing sequence of finite sets $\left\{C_{i} \mid C_{i}=C_{i}^{-1}\right\}$ and infinite sequence of elements $\left\{g_{i} \mid g_{i} \in C_{i} \backslash C_{i-1}^{5}\right\}$.

According to lemma 8 given $k$ one has $g_{j}^{-1} k g_{i} \notin C_{0}=H$ for all (except at most 2) pairs $(i, j), i \neq j$. Hence proximity (3) follows from (5) and the first conclusion of the claim is checked.

As regards the second conclusions of the claim we show that $\#\left\{i \mid g_{i}^{-1} k g_{i} \in H\right\} \leqslant 1$. In fact, if $g_{i}^{-1} k g_{i} \in H$ then for every $n>i$ one has $k \in I_{n}$ according to the choice of $I_{n}$. Since $g_{n} \in\left\{h_{j}\right\}$ it follows (from lemma 7) that $g_{n}^{-1} k g_{n} \notin H \backslash\{k\}$. Finally, $k \notin H \Leftarrow g \notin C$.

Now application of proximity in (5) finishes the proof.

\section{Construction of the SPECial $\mathcal{G}$-ACtion}

Fix two free mixing $\mathcal{G}$-actions $S: X \rightarrow X$ and $Q: Y \rightarrow Y$, collection of sets $r \subset \Sigma$, Rokhlin tower $\left\{Q^{g} E\right\}_{g \in G}^{\varepsilon}$, finite subsets $F,\left\{g_{i}\right\},\left\{c_{i}\right\}$ in $\mathcal{G}$, such, that $\sqcup_{i} F c_{i}=G$. Take an invertible measurepreserving mapping $V: X \mapsto X \times Y$ so that $A \times Y$ for every $A \in r$. Set $Z=V^{-1} J^{-1}(S \times Q) J V$ where the transformation $J$ is given by the formulas

$$
\left.J\right|_{X \times O}(x, y)=(x, y)
$$

and

$$
\left.J\right|_{X \times\left(Q^{f c_{i} E}\right)}(x, y)=\left(S^{f g_{i} f^{-1}} x, y\right)
$$

for all $c_{i}$ and all $f \in F$ (recall that $O$ denotes the remainder of the tower $\left\{Q^{g} E\right\}_{g \in G}^{\varepsilon}$ ).

$Z$ is called a special action.

Remark that for arbitrary $A, B \in r, g \in \mathcal{G}$ and an action $T$ one has

$$
\begin{gathered}
\left|\mu\left(T^{g} A \cap B\right)-\mu\left(V^{-1} J^{-1}\left(S^{g} \times Q^{g}\right) J V A \cap B\right)\right|= \\
=\left|\mu\left(T^{g} A \cap B\right)-\mu \otimes \mu\left(J^{-1}\left(S^{g} \times Q^{g}\right) J(A \times Y) \cap(B \times Y)\right)\right|= \\
=\mid \mu\left(T^{g} A \cap B\right)- \\
-\mu \otimes \mu\left(J^{-1}\left(S^{g} \times Q^{g}\right) J\left(A \times\left(\sqcup_{i, h} Q^{h c_{i}} E \sqcup O\right)\right) \cap\left(B \times\left(\sqcup_{j, f} Q^{f c_{j}} E \sqcup O\right)\right)\right) \mid \leqslant \\
\leqslant \sum_{f, h, i, j}\left|\mu\left(T^{g} A \cap B\right)-\mu\left(S^{g_{j}^{-1} f^{-1} g h g_{i} h^{-1}} A \cap S^{f^{-1}} B\right)\right| \mu\left(Q^{g h c_{i}} E \cap Q^{f c_{j}} E\right)+2 \mu(O) .
\end{gathered}
$$

Using notations (2), this inequality can be written in the form

$$
\begin{gathered}
\left|\mu\left(T^{g} A \cap B\right)-\mu\left(Z^{g} A \cap B\right)\right|< \\
<\sum_{f, h, i, j}\left|\mu\left(T^{g} A \cap B\right)-\mu\left(S^{g_{j}^{-1} k g_{i}} \tilde{A}_{h} \cap \tilde{B}_{f}\right)\right| \mu\left(Q^{g h c_{i}} E \cap Q^{f c_{j}} E\right)+2 \varepsilon
\end{gathered}
$$

The formula enables one to estimate the distance between $\mathcal{G}$-action $T$ and special action $Z$.

Theorem 3. Let $\mathcal{G}$ be finitely generated monotilable amenable group. For any free mixing $\mathcal{G}$-actions $S$ and $Q$ one has $\overline{S \times Q}=\mathcal{M}_{\mathcal{G}}$.

Proof. Given $\mathcal{G}$-action $T$ and positive number $\Delta$, we show that in $(m, \Delta)$-neighbourhood of $T$ there is an action conjugate to $S \times Q$.

First choose collection of measurable sets $r$ and $\varepsilon>0$ so that

$$
R \in \mathcal{Q}(T, r, 6 \varepsilon) \Rightarrow m(R, T)<\Delta
$$


Next fix a finite set $H \subset \mathcal{G}$ satisfying proximity

$$
\mu\left(T^{g} A \cap B\right) \stackrel{\varepsilon}{\sim} \mu(A) \mu(B)
$$

for all $A, B \in r, g \notin H$ and choose a tile $F$ so that $\#(g F \triangle F)<\varepsilon \# F$ for $g \in H$.

Passing to conjugate action one can assume that

$$
S^{g} \in \mathcal{U}\left(T^{g}, r, \frac{\varepsilon}{2}\right)
$$

for every $g \in F$ (see remark after lemma 5).

Using the previous claim choose corresponding elements $\left\{g_{i}\right\}$ and set $C$. Next by $Q, F, C$ and $\varepsilon$ choose sets $G \subset \mathcal{G}, E \subset Y$ and sequence $\left\{c_{i}\right\}$ as in lemma 6 .

Let $Z$ be the special action corresponding to the parameters obtained above. To show that $Z \in$ $\mathcal{Q}(T, r, 6 \varepsilon)$ we use inequality ([6) .

Given $A, B \in r$ and $g \in \mathcal{G}$ let a quadruple $(i, j, f, h)$ be called 'bad' if

$$
\left|\mu\left(T^{g} A \cap B\right)-\mu\left(S^{g_{j}^{-1} k g_{i}} \tilde{A}_{h} \cap \tilde{B}_{f}\right)\right|>2 \varepsilon
$$

(remember notations $k, \tilde{A}_{h}, \tilde{B}_{f}$ ). The points of $Q^{g h c_{i}} E \cap Q^{f c_{j}} E$ are called 'bad' if the corresponding quadruple $(i, j, f, h)$ is 'bad'.

We now estimates the measure of 'bad' points in the cases: $g \in H ; g \in C \backslash H ; g \notin C$.

1. Let $g \in H$. If in the first case $h, g h \in F$ then the set $Q^{g h c_{i}}$ is of the form $Q^{f c_{j}}$ hence $i=j$, $f=g h$. Since $k=e$ one has

$$
\begin{gathered}
\mu\left(S^{g_{j}^{-1} k g_{i}} \tilde{A}_{h} \cap \tilde{B}_{f}\right)=\mu\left(\tilde{A}_{h} \cap \tilde{B}_{f}\right)= \\
=\mu\left(S^{f h^{-1}} A \cap B\right)=\mu\left(S^{g} A \cap B\right) \stackrel{\varepsilon}{\sim} \mu\left(T^{g} A \cap B\right) .
\end{gathered}
$$

Thus, only the points of those $Q^{g h c_{i}} E \cap Q^{f c_{j}} E$ may be 'bad' for which $g h \notin F$. Measure of corresponding set does not exceed the ratio $\frac{\#(g F \triangle F)}{\# F}$ which is less than $\varepsilon$ by the choice of $F$.

2. Let $g \in C \backslash H$. If a quadruple $(i, j, f, h)$ is such that $h, g h \in F$ then all the points of $Q^{g h c_{i}} E \cap Q^{f c_{j}} E$ are not 'bad' (as above). Given a quadruple $(i, j, f, h)$ such that $h \in F, g h c_{i} \in G \backslash F c_{i}$ one has $i \neq j$ and according to claim 1 given $f, h$ and all pairs $(i, j)$, except at most 2

$$
\begin{gathered}
\left|\mu\left(S^{g_{j}^{-1} k g_{i}} \tilde{A}_{h} \cap \tilde{B}_{f}\right)-\mu\left(T^{g} A \cap B\right)\right| \leqslant \\
\leqslant\left|\mu\left(S^{g_{j}^{-1} k g_{i}} \tilde{A}_{h} \cap \tilde{B}_{f}\right)-\mu(A) \mu(B)\right|+\left|\mu(A) \mu(B)-\mu\left(T^{g} A \cap B\right)\right|<2 \varepsilon .
\end{gathered}
$$

Thus, only the points of those $Q^{g h c_{i}} E \cap Q^{f c_{j}} E$ may be 'bad' for which either $g h c_{i} \notin G$ or the pairs $(i, j)$ are exceptional. In the first case measure of corresponding set does not exceed the ratio $\frac{\#(g G \triangle G)}{\# G}$ which is less than $\varepsilon$ according to conclusion 2 of lemma 6. In the second case measure of the set of 'bad' points does not exceed

$$
\sum_{f, h} \sum_{(i, j) \in J} \mu\left(Q^{g h c_{i}} E \cap Q^{f c_{j}} E\right),
$$

where $J=J(f, h)$ is the set of exceptional pairs $(i, j)$. Since $\# J \leqslant 2$ this measure can be estimated as follows

$$
\sum_{f, h} 2 \mu(E)<\frac{2(\# F)^{2}}{\# G}<\varepsilon .
$$

3. Assume that $g \notin C$. According to claim for fixed $f, h$ and all pairs $(i, j)$, except at most 3 one has

$$
\begin{gathered}
\left|\mu\left(S^{g_{j}^{-1} k g_{i}} \tilde{A}_{h} \cap \tilde{B}_{f}\right)-\mu\left(T^{g} A \cap B\right)\right| \leqslant \\
\leqslant\left|\mu\left(S^{g_{j}^{-1} k g_{i}} \tilde{A}_{h} \cap \tilde{B}_{f}\right)-\mu(A) \mu(B)\right|+\left|\mu(A) \mu(B)-\mu\left(T^{g} A \cap B\right)\right|<2 \varepsilon
\end{gathered}
$$


Hence the points of $Q^{g h c_{i}} E \cap Q^{f c_{j}} E$ are not 'bad'.

Measure of the set of 'bad' points in this case does not exceed

$$
\sum_{f, h} \sum_{(i, j) \in J} \mu\left(Q^{g h c_{i}} E \cap Q^{f c_{j}} E\right)
$$

where $J=J(f, h)$ is the set of exceptional pairs. Since $\# J \leqslant 3$, for the total measure of 'bad' points can be estimated by

$$
\sum_{f, h} 3 \mu(E)<\frac{3(\# F)^{2}}{\# G}<\varepsilon .
$$

So the right hand side in (6) does not exceed the total measure of 'bad' points plus $2 \varepsilon$ one has that for all $g$

does not exceed $6 \varepsilon$.

$$
\left|\mu\left(T^{g} A \cap B\right)-\mu\left(Z^{g} A \cap B\right)\right|
$$

Corollary 2. Let $\mathcal{G}$ be finitely generated infinite monotilable group. Then for every Bernoulli action of this group conjugates are everywhere dense in $\mathcal{M}_{\mathcal{G}}$.

In fact theorem 3 can be applied since Bernoulli $\mathcal{G}$-action are Cartesian squares (see [2]).

Corollary 3. Let $\mathcal{G}$ be finitely generated infinite monotilable group. For every free mixing $\mathcal{G}$-action of positive entropy its conjugates are everywhere dense in $\mathcal{M}_{\mathcal{G}}$.

It follows from lemma 4 and the fact that free $\mathcal{G}$-action of positive entropy possesses a Bernoulli factor [2].

\section{Concluding Remark}

1. Resides weak and lead metrics in the set of $\mathcal{G}$-actions one can introduce mixed metrics. For example, metrics

$$
d(T, S)+\sup _{n \in \mathbb{N}} a\left(T^{n g}, S^{n g}\right)
$$

is complete and separable when considered in the set of $\mathcal{G}$-actions $T$ with mixing $T^{g}$. Such a metric was used in [3] for constructing of mixing transformations of homogeneous spectral multiplicity $n>2$.

2. The lead metrics may be introduced for actions of locally compact groups. In the case

$$
m(T, S)=\sum_{i} \frac{1}{2^{i}} \sup _{g \in K_{i}} d\left(T^{g}, S^{g}\right)+\sup _{g \in \mathcal{G}} a\left(T^{g}, S^{g}\right),
$$

where $\left\{K_{i}\right\}$ is a increasing to $\mathcal{G}$ sequence of compact sets.

\section{REFERENCES}

[1] S. V. Tikhonov, "A complete metric in the set of mixing transformations", Mat. Sb 198:4 (2007), 135-158; English transl. in Sb. Math. 198:4 (2007), 575-596.

[2] D. S. Ornstein and B. Weiss, Entropy and isomorphism theorems for actions of amenable groups. J. Analyse Math 48 (1987), 1-141.

[3] S. V. Tikhonov, Mixing transformations with homogeneous spectrum. Sbornik: Mathematics, 202(2011), No.8, 1231-1252

E-mail address: tikhonovc@mail.ru 